\title{
Sur une myxosporidiose de la thonnine (Euthynnus Alleteratus)
}

\author{
par J. F. ALDRIN
}

En août 1962, une thonnine pêchée dans les eaux du Golfe de Guinée fut apportée (malheureusement étêtée et vidée) au laboratoire du service des pêches maritimes par un armateur d'Abidjan, intrigué par les lésions internes que présentait ce poisson.

En effet, ainsi que le montre la photo no 1 la chair de ce thurnidae était truffée de petits kystes donnant au poisson un caractère répugnant.

\section{Description des lésions}

Les kystes ont en général une forme ovoïde et une coloration blanchâtre, leurs dimensions varient de 1 à 3 millimètres environ dans leur plus grande longueur. Ils sont parfois réunis en véritables amas.

Les kystes sont répartis dans la musculature et également dans le tissu conjonctif sous-cutané et sous-péritonéal. La concentration en est particulièrement forte dans le tissu conjonctif situé sous les nageoires dorsales et, à ce niveau, il est possible d'apercevoir les kystes par transparence à travers la peau. C'est d'ailleurs le seul endroit où les lésions sont visibles de l'extérieur.

D'une manière générale, les kystes sont surtout nombreux dans la partie postérieure du corps.

Macroscopiquement, la musculature ne semble pas altérée, sa coloration ef sa consistance sont normales.

La section des kystes révèle l'existence de deux zones, une zone périphérique, formant le kyste proprement dit, dont la consistance est fibreuse, et une zone médullaire, formée d'un liquide onctueux, laiteux, parfois densifié au point de former une véritable pâte.

Rev. Elev. Méd. vét. Pays trop. 1962, 15, no 4. Reçu pour publication : novembre 1962.

\section{Examen microscopique}

A l'examen microscopique du contenu des kystes, on constate que ce dernier est formé d'une véritable purée de spores de Myxosporidies (photo 2).

L'examentdirect sous lamelle, à l'immersion, permet d'a précier avec exactitude la forme de ces spores êt leurs dimensions. La forme, grossièrement sub-sphérique, est caractéristique et le schéma de la page suivante permet mieux qu'un texte de la décrire.

Ces dimensions sont les suivantes:

Plus grand diamètre: 10 microns environ.

Plus petit diamètre : 7 microns environ.

Après coloration au May-Grumwald et Giemsa, la structure de la spore apparaît nettement (photo no 3), elle possède quatre capsules polaires ovoïdes égales entre elles, groupées à une extrémité de la spore. Ces capsules ont environ 3 microns de longueur, sur 2 microns dans leur plus grande largeur.

Un certain nombre de capsules ont leur filament spiral dévaginé, mais il n'a pas été possible d'en apprécier la longueur avec exactitude.

En somme, la forme de cette spore et la présence de 4 capsules polaires permettent d'assigner à ce protozoaire une place: précise dans la nomenclature. Il s'agit d'une Myxosporidie appartenant au sous-ordre des sphaerosporea à la famille des Chloromyxidae et au genre Chloromyxum.

S'agit-il d'une espèce nouvelle? Il est difficile avec les moyens dont nous disposons ici de l'affirmer. En effet, des Chloromyxum ont déjà été signalés comme parasites musculaires d'un certain nombre d'espèces de poissons, tant d'eau douce que d'eau de mer, en particulier chez le maçuereau, par PÉRARD, chez le merlu de Mauritanie, par FLETCHER, HODGKISS et SHEWAN, et chez le thyrsite, par GILCHRIST. 


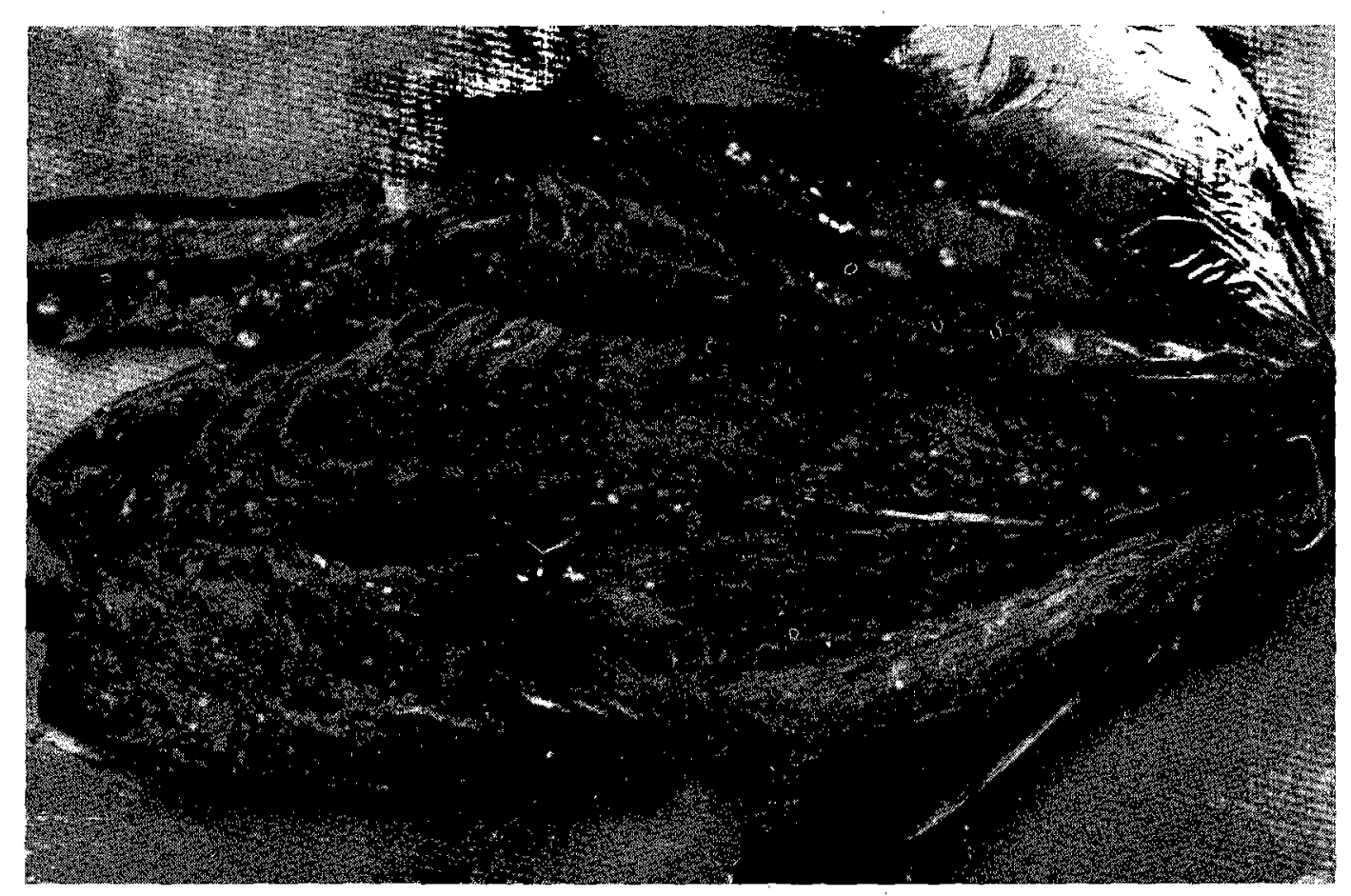

Photo no 1. - Aspect macroscopique des lésions.

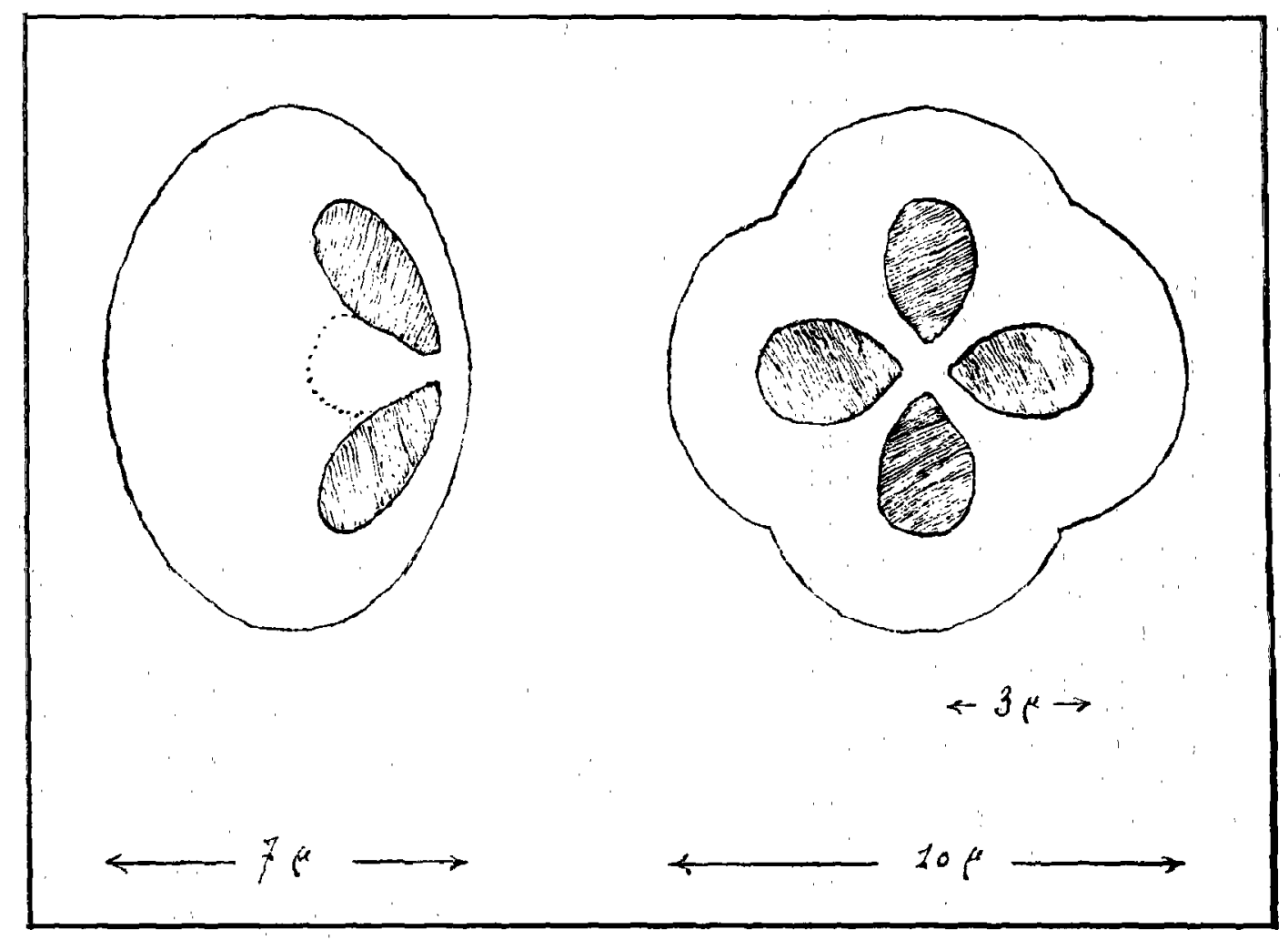

Spore de Chloromyxum sp. (schéma). 


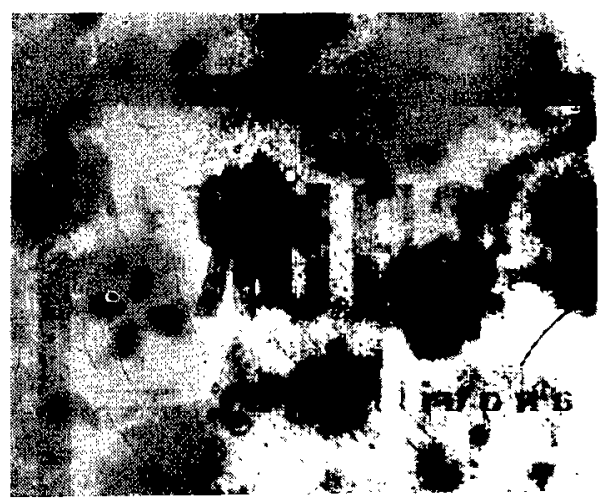

Photo no 2

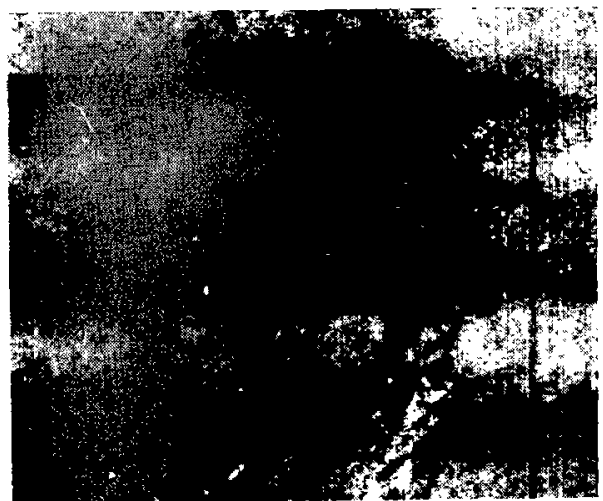

Photo no 3

Aspect nicroszopique des lésions.

Mais, dans tous ces cas, le signe essentiel de la maladie était un ramollissement et une dégénérescence de la chair musculaire. Au contraire, notre parasite n'entraîne upparemment aucune dégénérescence musculaire mais des formations kystiques localisées.

Il est difficile d'apprécier la fréquence de cette parasitose, car, sauf en cas d'infestation importante, elle peut très bien passer inaperçue. Tout au plus peut-on dire que c'est la première fois que nous la constatons ici, bien que de nombreuses thonnines soient pêchées dans les eaux ivoiriennes.

Par ailleurs, cette maladie de la thonnine ne semble pas avoir été décrite jusqu'à ce jour.

Service des pêches maritimes Abidjan (Côte d'Ivoire).

\section{RÉSUMÉ}

L'auteur décrit des lésions remarquées sur une thonnine et qu'il rapporte à une myxosporidiose. L'aspect de la spore et la présence de 4 capsules polaires font rattacher ce parasite au genre Chloromyxum.

\section{SUMMARY}

\section{Myxosporidiosis of the tunny-fish}

The lesions attributed to myxosporidiosis found in a tunny-fish are described. The nature of the spore with 4 polar capsules indicates its inclusion in the family of Chloromyxum.

\section{RESUMEN}

\section{Sobre una mixosporidiosis de la thonnina}

El autor describe las lesiones observadas sobre una thonnina y que él establece su correlación con una mixosporidiosis. El aspecto del esporo y la presencia de cuatro cápsulas polares hacen adscribir este parásito al género Chloromyxum. 